\title{
Enhanced detection of neoantigen- reactive $T$ cells targeting unique and shared oncogenes for personalized cancer immunotherapy
}

Rami Yossef, ${ }^{1}$ Eric Tran,, ${ }^{1,2}$ Drew C. Deniger, ${ }^{1}$ Alena Gros, ${ }^{1,3}$ Anna Pasetto, ${ }^{1}$ Maria R. Parkhurst, ${ }^{1}$ Jared J. Gartner, ${ }^{1}$ Todd D. Prickett, ${ }^{1}$ Gal Cafri, ${ }^{1}$ Paul F. Robbins, ${ }^{1}$ and Steven A. Rosenberg'

${ }^{1}$ Surgery Branch, National Cancer Institute, NIH, Bethesda, Maryland, USA. ${ }^{2}$ Earle A. Chiles Research Institute and the Providence Portland Medical Center, Portland, Oregon, USA. ${ }^{3}$ Medical Oncology Department, Vall d'Hebron University Hospital, Vall d'Hebron Institute of Oncology (VHIO), Pg. Vall d'Hebron, Barcelona, Spain.

\begin{abstract}
Adoptive cell transfer (ACT) of tumor-infiltrating lymphocytes (TILs) targeting neoantigens can mediate tumor regression in selected patients with metastatic epithelial cancer. However, effectively identifying and harnessing neoantigen-reactive $T$ cells for patient treatment remains a challenge and it is unknown whether current methods to detect neoantigen-reactive T cells are missing potentially clinically relevant neoantigen reactivities. We thus investigated whether the detection of neoantigen-reactive TILs could be enhanced by enriching T cells that express PD-1 and/ or T cell activation markers followed by microwell culturing to avoid overgrowth of nonreactive $T$ cells. In 6 patients with metastatic epithelial cancer, this method led to the detection of CD4+ and CD8 ${ }^{+}$cells targeting 18 and 1 neoantigens, respectively, compared with 6 and 2 neoantigens recognized by $\mathrm{CD}^{+}$and $\mathrm{CD}^{+}{ }^{+}$cells, respectively, when using our standard TIL fragment screening approach. In 2 patients, no recognition of mutated peptides was observed using our conventional screen, while our high-throughput approach led to the identification of 5 neoantigen-reactive $T$ cell receptors (TCRs) against 5 different mutations from one patient and a highly potent MHC class II-restricted KRAS ${ }^{\mathrm{C} 12 \mathrm{~V}}$-reactive TCR from a second patient. In addition, in a metastatic tumor sample from a patient with serous ovarian cancer, we isolated 3 MHC class II-restricted TCRs targeting the $\mathrm{TP}^{2}{ }^{\mathrm{C} 2455}$ hot-spot mutation. In conclusion, this approach provides a highly sensitive platform to isolate clinically relevant neoantigen-reactive T cells or their TCRs for cancer treatment.
\end{abstract}

Conflict of interest: The authors have filed patent applications related to KRASC12V (US application no. 62/560,930) and TP53G245S (US application no. 62/565,383).

Submitted: May 24, 2018 Accepted: August 21, 2018 Published: October 4, 2018

\section{Reference information:} JCI Insight. 2018;3(19): e122467. https://doi.org/10.1172/jci. insight.122467.

\section{Introduction}

Tumor cells acquire nonsynonymous mutations during tumorigenesis (1). Some nonsynonymous mutations, denoted as neoantigens, can trigger $\mathrm{T}$ cell activation via $\mathrm{T}$ cell receptor (TCR) engagement with MHC molecules (HLA, in human) presenting the peptide containing the mutated amino acid (pHLA) on target cells (2). Recently, we showed in 101 metastatic melanoma patients that reinfusing autologous tumor-infiltrating lymphocytes (TILs) could mediate a durable complete response (CR) rate and overall response rate (ORR) of $24 \%$ and $56 \%$, respectively (3). Furthermore, adoptive cell transfer (ACT) treatment with selected TILs targeting neoantigens caused significant tumor regression in a small percentage of metastatic epithelial cancer patients (4-6). One potential explanation for this low response is that the $\mathrm{T}$ cells used for treatment contained a low frequency of tumor-reactive $\mathrm{T}$ cells, which may be a consequence of the overgrowth of nonreactive cells during extensive in vitro culturing of $\mathrm{T}$ cells that is required to generate sufficient $\mathrm{T}$ cell numbers for patient treatment. Due to persistent antigen exposure in the tumor microenvironment, TILs often present exhaustion hallmarks and therefore their growth in vitro can be limited (7). In addition, due to intratumoral heterogeneity, neoantigens targeted by ACT can be expressed subclonally in the tumor, resulting in tumor escape $(8,9)$. To address these issues, we are planning a new clinical trial in which we retrovirally transduce autologous peripheral blood mononuclear cells (PBMCs) with neoantigenreactive TCRs isolated from the patient (NCT03412877). Targeting multiple neoantigens or shared oncogenes may increase our chances of attacking multiple clones of the tumor. 
To select TILs for treatment as part of clinical trial NCT01174121, we cut excised tumor(s) into 1- to $2-\mathrm{mm}^{3}$ fragments, cultured them ex vivo in high-dose IL-2, and tested the TILs from the fragments for reactivity against neoantigens using peptides or tandem minigenes (TMGs) encompassing the mutations identified in the tumor. TIL cultures that contain reactive $\mathrm{T}$ cells are further expanded and reinfused to the patient $(10,11)$. Although several techniques to enrich for reactive T cells have been developed (12-18), the detection of tumor- or neoantigen-reactive $\mathrm{T}$ cells is still challenging. In a number of these methods, $\mathrm{T}$ cells are enriched based on surface marker expression and further expanded and tested $(15,16,18-20)$. Gros et al. and others showed that sorting TILs expressing programmed cell death protein 1 (PD-1) enriched tumor- and neoantigen-reactive $\mathrm{T}$ cells in patients with melanoma. However, several studies showed that terminally differentiated effector memory cells (Temra) express low levels of PD-1 on their cell surface (21, 22), although these cells were chronically exposed to antigen and may be clinically relevant (23). CD137, a costimulatory $\mathrm{T}$ cell protein, was also suggested as a candidate for reactive $\mathrm{T}$ cell enrichment; nevertheless, while CD137 is expressed on activated $\mathrm{CD} 8^{+}$cells it may not be reliable, as a sole marker, for the enrichment of activated $C D 4^{+}$cells $(15,24)$. Importantly, in these enrichment approaches, the cells are expanded as mixed cultures that could lead to overgrowth by younger, potentially nonreactive cells, thereby diluting the reactive, exhausted cells in the culture $(13,16-18)$. An additional method isolated $\mathrm{T}$ cells from tumor and blood using pHLA tetramers, but this method was restricted to $\mathrm{CD} 8^{+}$cells and relied on predictions of peptide-HLA binding (12)

In the present study, we developed a nonbiased, 2-step, high-throughput limiting-dilution approach for detection and isolation of neoantigen-reactive $\mathrm{T}$ cells that can potentially overcome some of the problems discussed above. This approach enabled the enhanced detection of neoantigen-reactive TILs in metastatic nonmelanoma tumor samples and the rapid isolation of neoantigen-reactive TCRs for potential use in clinical trials. In this method, we FACS isolated TILs that expressed T cell costimulatory markers (CD134 or CD137) and/or PD-1. We reasoned that the use of both markers would enhance the enrichment of neoantigen-reactive $\mathrm{CD} 4^{+}$cells that would otherwise be missed by enriching TILs using CD137 alone and Temra cells that express low levels of PD-1. Sorted cells underwent limiting-dilution cloning and were then expanded in microwell plates to avoid overgrowth of nonreactive young cells at the expense of exhausted reactive cells. Cultures that grew were tested against 25 mer peptides encompassing mutations that were present in patient tumors. Employing this approach enabled us to detect 19 neoantigen $\mathrm{T}$ cell reactivities in 6 epithelial cancer samples. Moreover, $\mathrm{CD} 4^{+} \mathrm{T}$ cells reactive against $\mathrm{KRAS}^{\mathrm{G} 12 \mathrm{~V}}$ and $\mathrm{TP} 53^{\mathrm{G} 245 \mathrm{~S}}$ hot-spot mutations were isolated from 2 patients. Notably, reactive cultures were highly oligoclonal or clonal, which allowed us to efficiently sequence reactive TCRs using single-cell PCR (scPCR). This strategy provides a sensitive approach for the enhanced detection of neoantigen-reactive TILs and can be utilized for the rapid identification of neoantigen-reactive TCRs, which can be used to generate a personalized, neoantigentargeted TCR-gene therapy for patients with cancer.

\section{Results}

Developing a high-throughput TIL microwell culture method to detect and isolate neoantigen-reactive TCRs. The scheme we used to isolate neoantigen-reactive TILs is shown in Figure 1. Metastatic lesions were excised from cancer patients, enzymatically digested to obtain a single-cell suspension, and incubated overnight in T cell medium without cytokines. Cells were then stained and T cells were FACS isolated based on the expression of PD-1 and/or T cell activation markers (single positives and double positive, Figure 1). Enriched cells were cultured at low concentration in rapid expansion protocol (REP) conditions in the presence of irradiated allogeneic feeder cells, IL-2, and OKT3 antibody, as described in detail in the Methods (Figure 1). Three to 4 weeks later, neoantigen reactivity of the expanded cells was tested based on IFN- $\gamma$ secretion and expression of T cell activation markers after coculture with autologous antigen-presenting cells (APCs) expressing the patient's own neoantigens as described in the Methods. Briefly, to decrease the number of microcultures screened, cells from 2 cultures that grew were combined and cocultured with patient's DCs pulsed with 2 pools of $25 \mathrm{mer}$ peptides containing the nonsynonymous mutations expressed in the patient's tumor flanked by 12 amino acids of the wild-type (WT) protein (Figure 1 and ref. 25). Cultures that showed specific reactivity against the pools, defined by 2 -fold higher IFN- $\gamma$ secretion or upregulation of activation markers compared with the negative controls, were further expanded for testing (Figure 1). Additionally, cells from coculture wells that showed reactivity against peptide pools in IFN- $\gamma$ ELISPOT assays were collected, stained, and FACS isolated based on activation markers in 96-well plates for single-cell TCR sequencing (Figure 1). 


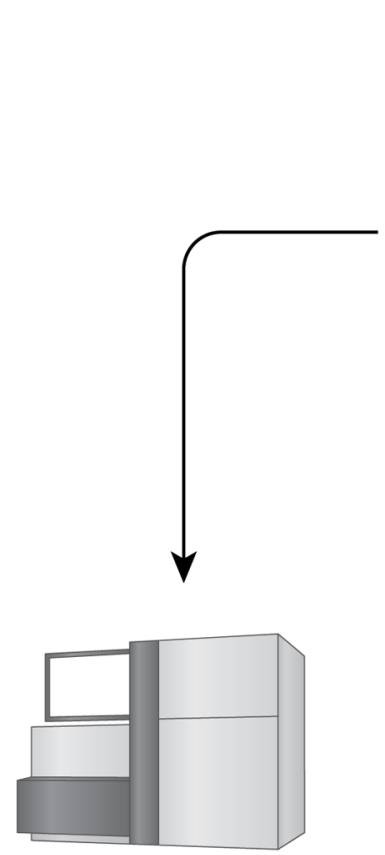

2a. WES and RNAseq

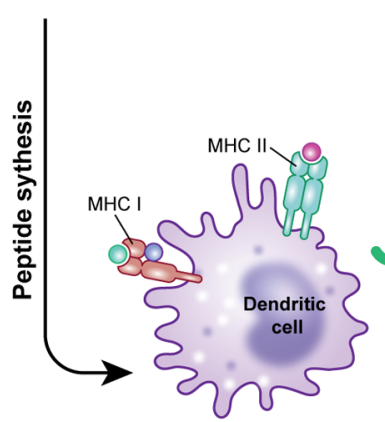

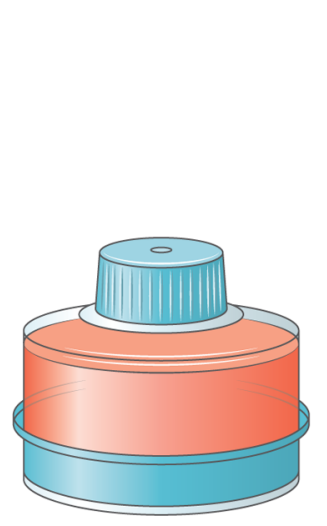

5a. REP for positive wells $\&$ further screening
4. First screen against pooled peptide pools $(\sim 3$ weeks)
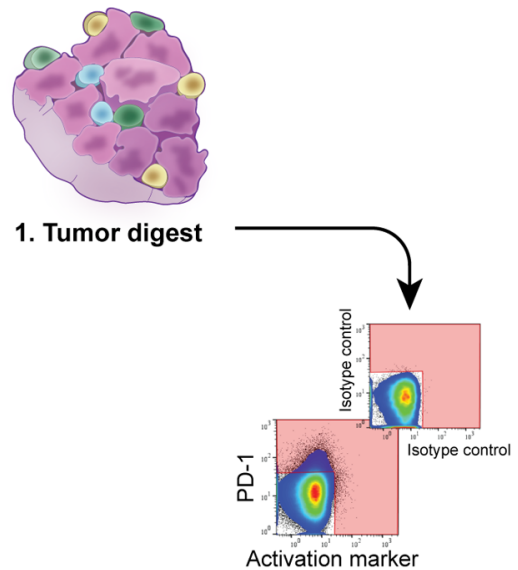

2b. Sorting $T$ cells expressing PD-1+ and/or activation marker

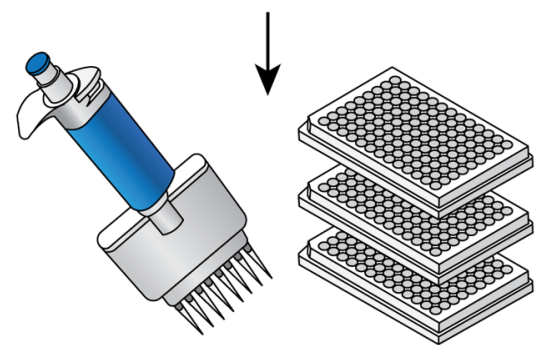

3. Culturing sorted $\mathrm{T}$ cells in limiting dilution conditions + irradiated feeder cells
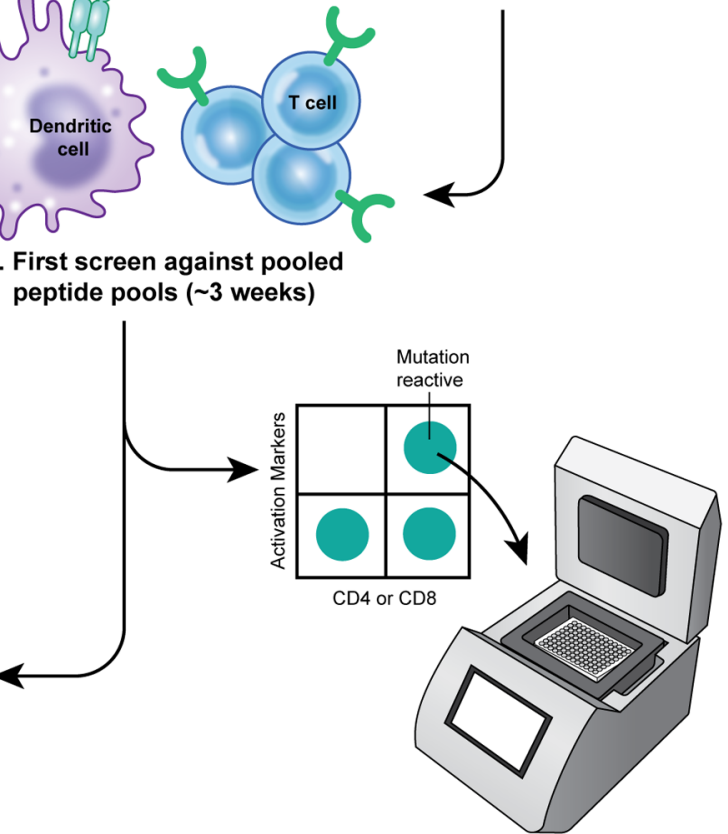

5b. ScPCR to isolate mutation-reactive TCRs
Figure 1. Illustration of the new high-throughput approach for enrichment, culturing, and screening strategy of TILs. (1) Tumor cell digests were thawed and rested overnight in complete media in the absence of exogenous cytokines. (2a) A piece of the tumor underwent whole-exome sequencing (WES) and RNA sequencing to identify nonsynonymous mutations. Based on mutation calls, 25 mer peptides encompassing the mutations at position 13 were synthesized. (2b) Cells were washed, labeled, and sorted based on PD-1 and/or activation markers (CD134 or CD137) expression (pink area represents that gate used in the sort). (3) Sorted cells were cultured in 96-well plates at 3 cells/well in the presence of irradiated allogeneic feeder cells, 3,000 IU/ml IL-2, and anti-CD3 $\varepsilon$ (OKT3) for expansion. (4) Peptide pools were pulsed on autologous APCs that served as a target in a coculture with sorted cells that grow in the microwell cultures. To minimize the assays, cells from 2 or 3 cultures were combined in the assay wells. (5a) Cultures that showed recognition against peptide pools were further expanded for future testing. (5b) Cells from Pt.4097 coculture assay were labeled and reactive $T$ cells were single-cell sorted into 96-well plates containing lysis buffer and PCR primers for TCR sequencing.

Enhanced detection and isolation of neoantigen-reactive TILs in metastatic epithelial tumors. To test the feasibility and sensitivity of our high-throughput culturing approach to detect neoantigen-reactive TILs, we studied a metastatic lesion from the adrenal gland in a patient with metastatic gastroesophageal junction adenocarcinoma (Pt.4078). This patient was enrolled in the NCT01174121 study for possible treatment using ACT with neoantigen-reactive TILs. Neoantigen-reactive T cells were not detected in TILs when tumor fragments were screened using our standard method (Supplemental Figure 1A; supplemental material available online with this article; 
A
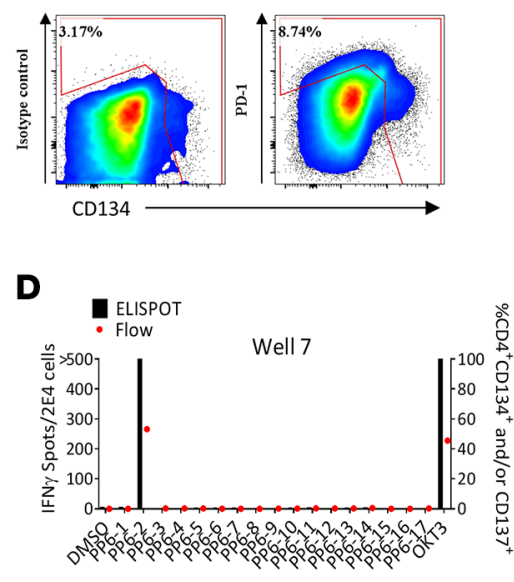

B

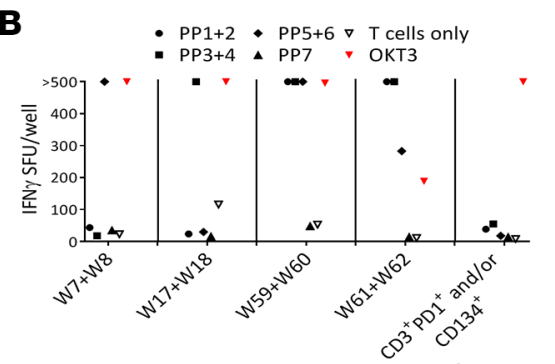

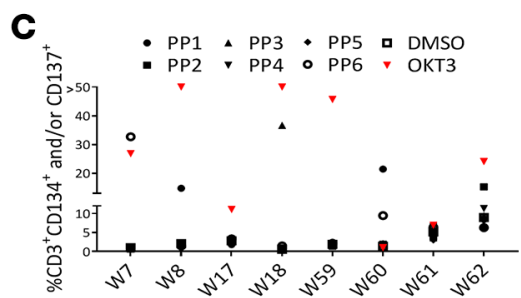
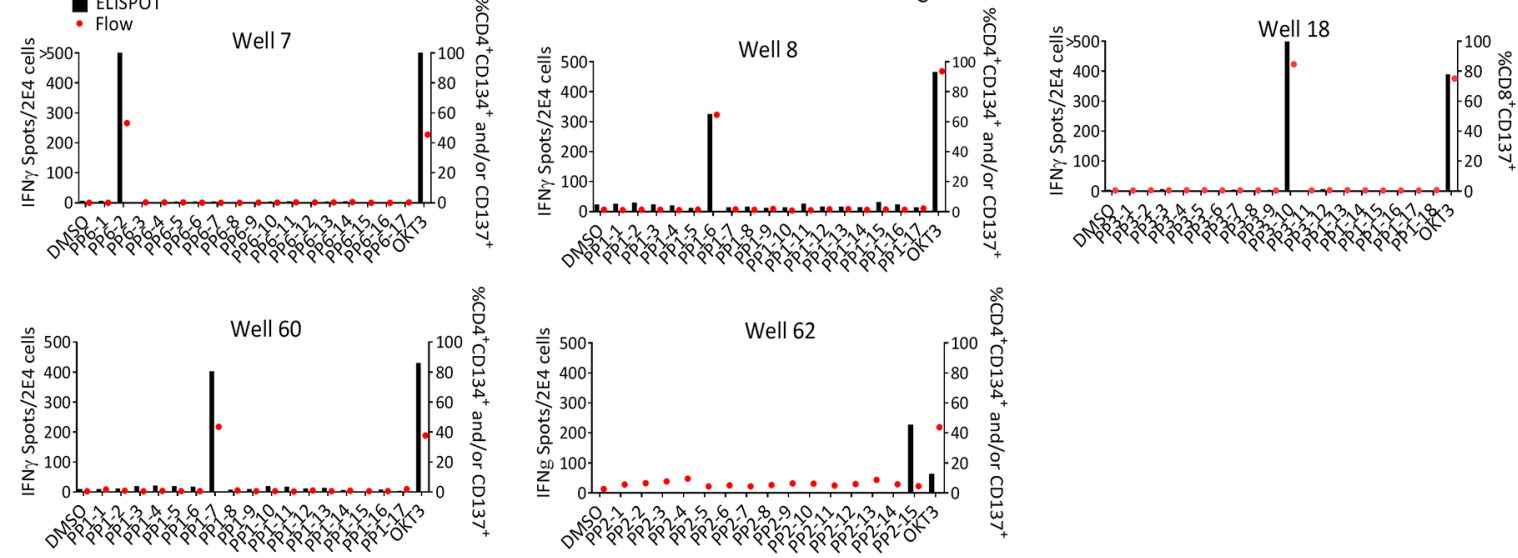

E
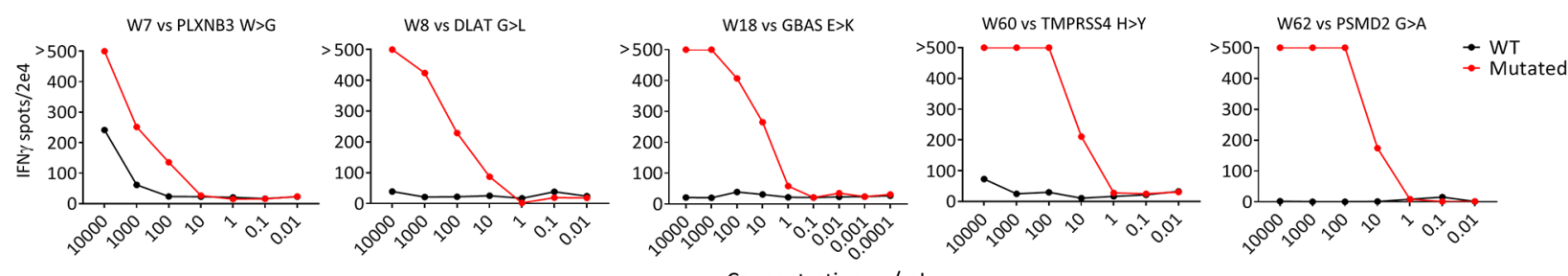

Figure 2. Sorting strategy, functional screen assays, and identification of neoantigen-reactive TCRs in Pt.4078. (A) Gating strategy used in flow cytometry-based sort for CD3+PD-1+ and/or CD134+ TILs. (B-D) ELISPOT assays measuring IFN- $\gamma$ secretion of microwell cultures upon coculture with target cells. (B) Following expansion, pools of 2 cultures were tested against autologous DCs pulsed with 2 peptide pools (PP), indicated by symbols. (C) Cultures from the reactive pools were tested separately against autologous DCs pulsed with all suspected peptide pools. (D) IFN- $\gamma$ ELISPOT and CD137 flow cytometry analysis showing reactivity of the TIL cultures following coculture with autologous DCs pulsed with single $25 \mathrm{mer}$ peptides from each peptide pool. (E) Allogeneic T cells retrovirally transduced with neoantigen-reactive TCRs cocultured with autologous DCs pulsed with serially diluted mutated and WT 25mer peptides. '>' denotes greater than 500 spots. All data are representative of at least 3 independent experiments except in $\mathbf{A}$.

https://doi.org/10.1172/jci.insight.122467DS1). We then used the frozen tumor digest as a source of TILs by FACS isolating the $\mathrm{CD} 3^{+} \mathrm{PD}-1^{+}$and/or $\mathrm{CD} 3^{+} \mathrm{CD} 134^{+}$subset (Figure $\left.2 \mathrm{~A}\right)$. Cells $\left(1.5 \times 10^{3}\right)$ were sorted, diluted to 3 cells/well, and cultured in REP conditions. Three weeks later, 64 cultures, labeled W1-W64, grew ( 13\% growth efficiency) and were tested for reactivity against 104 25mer peptides grouped in 7 peptide pools (PP1 through PP7; Supplemental Table 3). Eight cultures showed substantial reactivity against several peptide pools (Figure 2B); however, the observation that TILs from W59 and W60 showed recognition of multiple peptide pools suggested that there was some peptide cross contamination across some peptide pools.

The 8 reactive cultures were further expanded using the REP and retested against PP1 through PP6 (Figure 2C). At this stage, the functional coculture assays showed that 5 cultures (W7, W8, W18, W60, and W62) recognized unique peptide pools. Interestingly, culture W8 showed specific IFN- $\gamma$ secretion when tested against PP1 that was not detected in the first screen, which might be attributable to insufficient peptide presentation on the DCs due to a large number of peptides tested in the first screen (30 peptides were used in the first screen; Figure 2B).

Next, we sought to identify the neoantigens recognized by microwell TIL cultures. To this end, we performed $\mathrm{T}$ cell cocultures against $\mathrm{DC}$ s pulsed with individual peptides derived from the relevant 
Table 1. TRBV sequences of neoantigen-reactive cultures and their frequency in tumor digest subsets

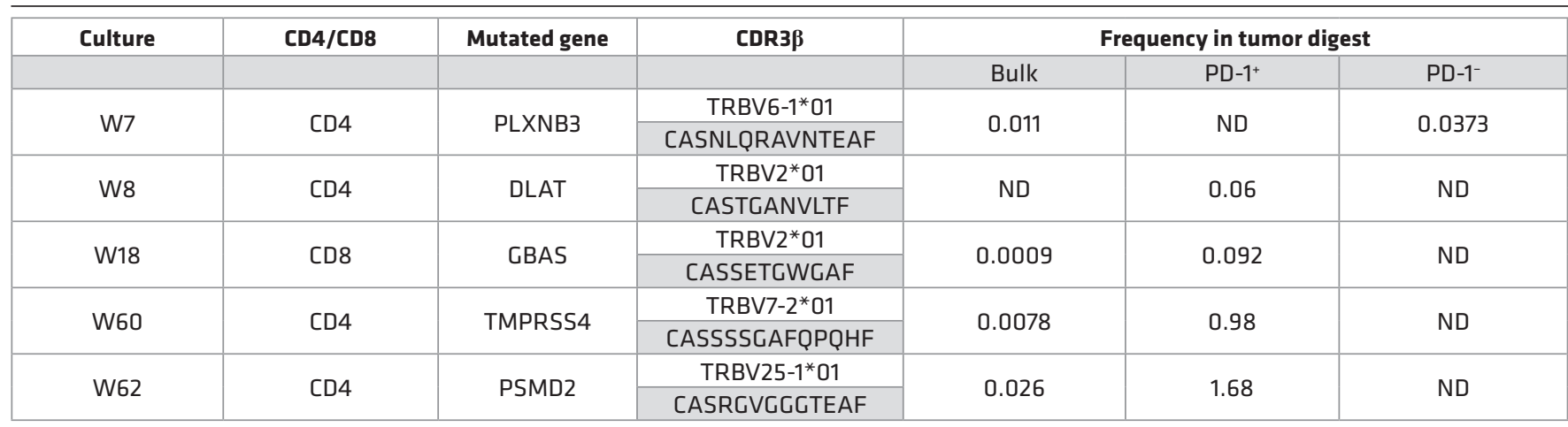

ND, not detected.

peptide pools (Figure 2D). Both IFN- $\gamma$ secretion and upregulation of T cell activation markers assessed by flow cytometry demonstrated that each TIL culture was reactive solely against 1 potential neoantigen. Also, by coculturing W18 with COS7 cells transfected with the predicted or control HLAs and pulsed with the predicted minimal epitope, we were able to determine the restriction of the reactivity (HLA-A*30:01, Supplemental Figure 1B). At this point, we were able to sequence TCR $\alpha$ and TCR $\beta$ of the reactive cultures using 5'RACE rapid cDNA amplification, using TCR $\alpha$ - and TCR $\beta$-specific primers, followed by Sanger sequencing. All 5 cultures displayed unique and clonal TCRs (Table 1). To verify that the sequences of the $\alpha$ and $\beta$ chain are indeed encoding for neoantigen-reactive TCRs, we first cloned the sequences into a retroviral vector (pMSGV1) and introduced the TCRs into allogeneic PBMCs, as described previously (13). Subsequently, coculture of the TCR-gene-modified cells with autologous APCs pulsed with a serial dilution of HPLC-grade mutated and counterpart WT peptides confirmed that the isolated TCRs were neoantigen reactive (Figure 2E).

To evaluate the sensitivity of our new high-throughput approach we determined the frequencies of the neoantigen-reactive TCR $\beta$ sequences in the tumor digest. For this purpose, we performed TCR $\beta$ deep sequencing of the bulk tumor digest and 4 FACS-isolated subsets of the tumor digest: CD $4{ }^{+} \mathrm{PD}-1^{+}$, CD $4^{+} \mathrm{PD}-1^{-}, \mathrm{CD} 8^{+} \mathrm{PD}-1^{+}$, and $\mathrm{CD} 8^{+} \mathrm{PD}-1^{-}$. Frequencies of the neoantigen-reactive TCRs in the bulk population were extremely low, ranging from $0.026 \%$ to $0.0009 \%$ (Table 1 ), which may at least partially explain why no neoantigen reactivity was detected using the TIL fragment screen approach. Moreover, 4 out of the 5 TCRs were detected in the $\mathrm{PD}-1^{+}$subsets and only one, W7, was detected in the $\mathrm{PD}-1^{-}$subset, suggesting that it likely came from the $\mathrm{PD}-1^{-} \mathrm{CD} 134^{+}$population, which is consistent with our previous observations that sorting $\mathrm{PD}-1^{+} \mathrm{T}$ cells from tumor digests enriches for tumor- and neoantigen-reactive $\mathrm{T}$ cells (13). Nevertheless, in some cases like culture W7, enrichment for PD- $1^{+}$alone is insufficient to isolate all neoantigen-reactive $\mathrm{T}$ cells. However, this could be overcome by using other $\mathrm{T}$ cell activation markers, such as CD134. In summary, we were able to detect $\mathrm{T}$ cell reactivities against 5 neoantigens that were not detected when multiple TIL fragments from the same lesion were tested. Moreover, we were able to isolate rare neoantigen-reactive TCRs.

To assess the reproducibility of our approach, we employed our new high-throughput multiwell culturing protocol to screen 5 additional metastatic tumor digest samples from cancer patients with various histologies. All subjects were previously screened using the tumor fragment screening approach, as part of the NCT01174121 trial (Table 2 and Supplemental Table 1). Using our high-throughput multiwell culturing approach we were able to detect TIL reactivities in all 5 additional cases. In total, we were able to detect neoantigen reactivities against 19 different neoantigen targets as compared with 9 that were detected using TIL fragment screening (Table 2, Supplemental Table 2, and Supplemental Figures 2-5). In the new approach, $\mathrm{CD}^{+}$and $\mathrm{CD} 8^{+} \mathrm{T}$ cells targeting 18 and 1 neoantigens, respectively, were found, while 6 and 2 neoantigens recognized by CD4 ${ }^{+}$ and $\mathrm{CD}^{+} \mathrm{T}$ cells, respectively, were detected using the standard TIL fragment screening method. Out of the 6 neoantigen reactivities targeted by $\mathrm{CD} 4^{+} \mathrm{T}$ cells identified using the TIL fragment screening approach, 5 were identified in our new method. Overall, these results demonstrate the reproducibility and the high sensitivity of our new culturing and screening approach. 
Table 2. Summary of neoantigen reactivities found using the new approach compared with reactivities found in TIL fragments

\begin{tabular}{|c|c|c|c|c|c|c|c|c|}
\hline Patient ID & $\operatorname{Age}^{A} /$ Sex & Tumor histology & $\begin{array}{l}\text { No. of mutations } \\
\text { assessed }\end{array}$ & $\begin{array}{c}\text { Reactivities } \\
\text { found in TIL } \\
\text { fragment screen }\end{array}$ & T cell type & $\begin{array}{l}\text { Reactivities } \\
\text { found in LD } \\
\text { cultures }^{B}\end{array}$ & T cell type & $\begin{array}{l}\text { No. of reactive } \\
\text { TCRs found using } \\
\text { LDC }^{c}\end{array}$ \\
\hline \multirow[t]{4}{*}{4078} & $48 / M$ & $\begin{array}{l}\text { Gastroesophageal } \\
\text { junction } \\
\text { adenocarcinoma }\end{array}$ & 104 & None & & GBASE207K & CD8 & 1 \\
\hline & & & & & & PLXNB3 ${ }^{\text {W609C }}$ & $\mathrm{CD} 4$ & 1 \\
\hline & & & & & & DLAT $\mathrm{C}^{\mathrm{C} 94 \mathrm{~L}}$ & CD4 & 1 \\
\hline & & & & & & PSMD2 ${ }^{\mathrm{C} 644 \mathrm{~A}}$ & CD4 & 1 \\
\hline \multirow[t]{3}{*}{4097} & $59 / F$ & Ovarian & 317 & HIST1H1B A71D & CD4 & HIST1H1B A71D & CD4 & 7 \\
\hline & & & & INPP5K L176V & CD4 & HYAL4 ${ }^{\text {R945 }}$ & $\mathrm{CD} 4$ & 1 \\
\hline & & & & & & HSPG2 $2^{\mathrm{H} 3568 \mathrm{~L}}$ & $\mathrm{CD} 4$ & 1 \\
\hline 4148 & $68 / F$ & Endometrial & 108 & None & & KRASG12V & $\mathrm{CD} 4$ & 1 \\
\hline 4217 & $49 / M$ & Colon & 176 & MAP3K $2^{5153 F}$ & CD4 & MAPЗK $2^{5153 F}$ & $\mathrm{CD} 4$ & 1 \\
\hline \multirow[t]{4}{*}{4127} & $58 / F$ & Ovarian & 180 & TP53 $3^{\mathrm{G} 2455}$ & CD4 & TP53 $3^{\mathrm{G} 2455}$ & CD4 & 3 \\
\hline & & & & & & HIST1H2BM E77V & CD4 & 1 \\
\hline & & & & & & GORASP L248FS $\ddagger$ & CD4 & $3(2+1)$ \\
\hline & & & & & & TUBA1B $^{5287 T}$ & $\mathrm{CD} 4$ & 1 \\
\hline \multirow[t]{4}{*}{4166} & $40 / M$ & Pancreatic & 156 & NPLOC4 $4^{1312 V}$ & CD8 & ZNF727" ${ }^{\mathrm{H} 163 \mathrm{Q}}$ & CD4 & 1 \\
\hline & & & & & & TNC E743D & CD4 & 4 \\
\hline & & & Total: & 8 & CD8: 2 & 19 & CD8: 1 & \\
\hline & & & & & CD4: 6 & & CD4: 18 & \\
\hline
\end{tabular}

${ }^{A}$ At the time of admission. ${ }^{B}$ Neoantigen specificity was determined by testing against WT peptides. ${ }^{C}$ TCRs that were constructed and tested are bolded. ҒFS, frame-shift mutation. LD, limiting dilution.

Rapid identification and sequencing of neoantigen-reactive TIL TCRs. For metastatic cancer patients, the time to clinical intervention is critical. In our attempt to shorten the time of isolation of neoantigen-reactive TCRs, we utilized scPCR in our screening pipeline to detect reactive TILs, as previously described (15). To investigate the feasibility of incorporating this approach early in the microwell screens we studied cryopreserved cell digests of a metastatic right groin lymph node from a patient with serous ovarian carcinoma (Pt.4097). Following the in vitro expansion of microwell cultures containing $\mathrm{CD} 3^{+} \mathrm{PD}-1^{+}$and/or $\mathrm{CD} 134^{+}$cells, a functional assay was carried out, as described above (Figure 3A). Following the incubation coculture, we transferred the cells from the ELISPOT plate into new 96-well plates. After we developed the IFN- $\gamma$ ELISPOT assay plates we stained the cells from coculture wells that showed enhanced IFN- $\gamma$ secretion and sorted single T cells that upregulated $\mathrm{T}$ cell activation markers into 96-well PCR plates for TCR sequencing (Figure 3B). Several microwell TIL cultures showed recognition against PP15+16, while only 2 coculture wells showed recognition against DCs pulsed with PP1+2 or PP17+18, respectively. Similar to previous screens, for further study of the reactivities against mutated peptides, putative TIL microwell cultures were further expanded for 2 weeks. However, following the first coculture assay, we were able to obtain unique TCR sequences of the reactive wells with our SCPCR method combined with Sanger sequencing (Figure 3C). Next, following expansion, we studied the reactivities of the single TIL microwell cultures against APCs pulsed with single peptide pools and the individual peptides (Figure 3D). After we identified the neoantigens, we sequenced the TCRs of the reactive cultures using TCR $\beta$ deep sequencing and confirmed the sequences that were obtained using scPCR. In summary, we were able to identify and sequence neoantigen-reactive TCRs 4 weeks after we seeded the microwell TIL cultures. This rapid and reliable approach could be valuable for our clinical trial seeking to improve the efficacy of personalized cancer immunotherapy treatment with ACT using PBMCs genetically modified to express neoantigen-reactive TCRs.

Isolating TP53 hot-spot driver mutation-reactive TCRs. The overwhelming majority of cancer-associated mutations are private and unique to the individual, which means that the targeted neoantigens expressed in 
A

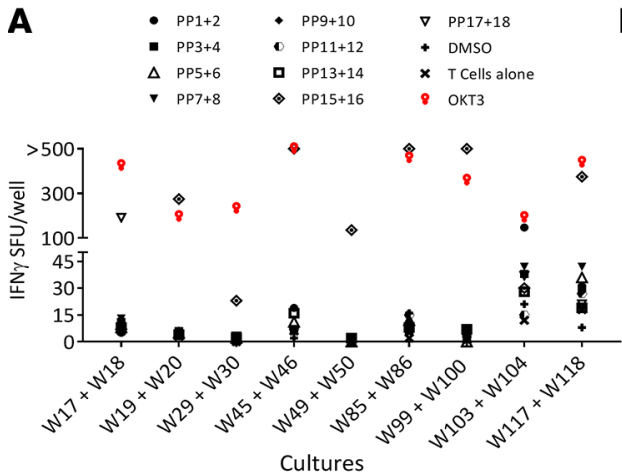

C

\begin{tabular}{|c|c|c|}
\hline Co-culture wells & & DR3 $\beta$ \\
\hline$W 17+W 18$ & TRBV5-1*01 & CASTRTSGGGETQYF \\
\hline$W_{19}+W_{20}$ & TRBV19*02 & CASTGTSNEKLFF \\
\hline$W 29+W 30$ & TRBV10-2*01 & CASSERQNSNQPQHF \\
\hline$w 45+W 46$ & TRBV28*01 & CASSPGGQNSPLHF $^{+}$ \\
\hline$W 49+W 50$ & TRBV6-5*01 & CASSRGPQRGETQYF \\
\hline W85 + W86 & TRBV4-2*01 & CASSLSLEQPQHF \\
\hline$W 99+W 100$ & TRBV12-3*01 & CASSGTANTGELFF \\
\hline W103 + W104 & TRBV7-7*01 & CASSLDIIGEQFF \\
\hline W117 + W118 & TRBV28*01 & CASSPGGQNSPLHF $^{+}$ \\
\hline
\end{tabular}

+ - Same clone

Bolded - The reactive wells. Based on functional assays of the individual wells

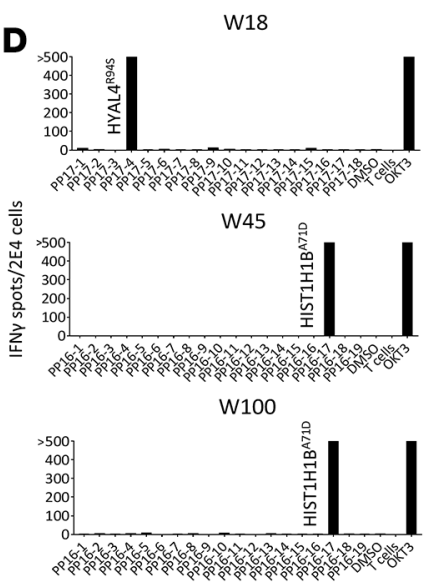

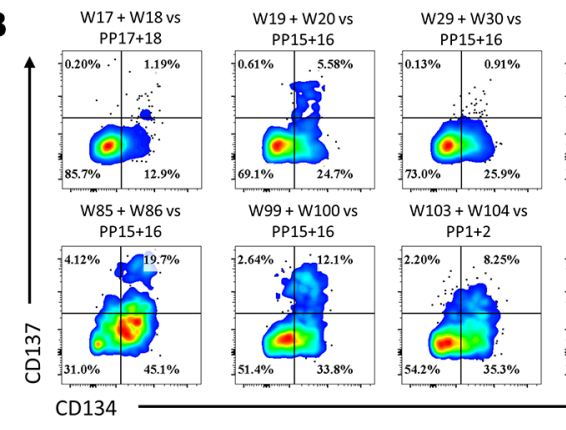
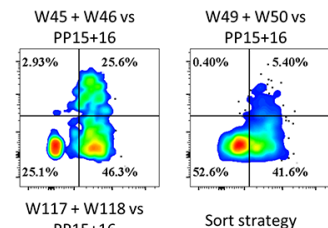

PP15+16

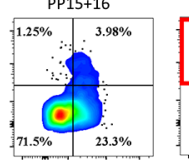

Sort strategy

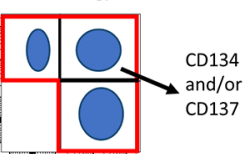

Gated on Live $\mathrm{CD}^{+} \mathrm{CD}^{+}$

134
d/or
CD137

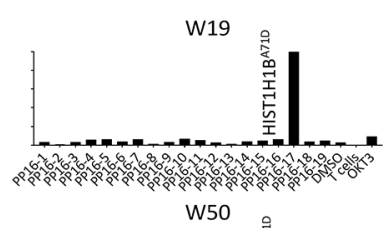

W50
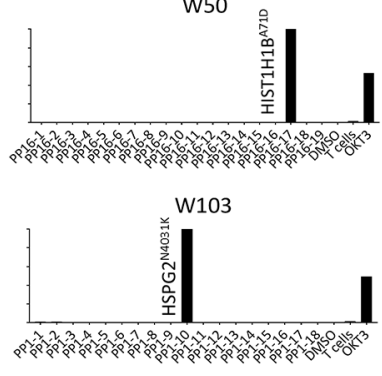

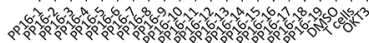

W85

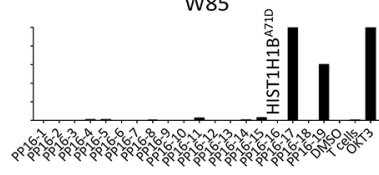

W30

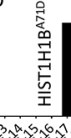

11

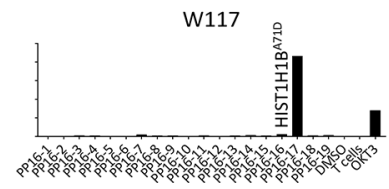

Figure 3. Rapid sequencing of neoantigen-reactive TCRs from Pt.4097. (A and B) Pools of TIL cultures incubated with DCs pulsed with pooled peptide pools (PP), indicated by symbols. (A) Summary of TIL culture pools showing secretion of IFN- $\gamma$ in ELISPOT. (B) Cells from ELISPOT coculture wells were collected, labeled, and single cells expressing T cell activation markers were sorted (as shown in the bottom right panel) into a 96-well PCR plate containing lysis buffer and PCR primers for TCR $\alpha$ and - $\beta$. (C) CDR3 $\beta$ of the sequenced culture well. Bolded are the origin of the reactive TCR, based on functional assays done with the individual cultures (data not shown). (D) Expanded cultures from the indicated TIL microwells coincubated with autologous DCs pulsed with single peptides from the pools that the cultures showed recognition against in previous experiments, representative of at least 2 independent experiments. '>' denotes greater than 500 spots.

one patient are not likely to be expressed in other patients. Moreover, due to intratumoral heterogeneity, private mutations might be subclonal. On the other hand, public or shared hot-spot mutations are more likely to be expressed by all cells within a tumor as well as across tumor samples and histologies. Thus, ACT using off-the-shelf TCRs targeting shared mutations when possible appears to be an additional attractive strategy to target cancer neoantigens.

To this end, we employed our approach to screen for neoantigen reactivities in Pt.4127, a metastatic ovarian cancer patient whose tumor expressed a hot-spot mutation in the TP53 gene. In a previous study, CD4 ${ }^{+}$ $\mathrm{T}$ cells reactive to the TP53 ${ }^{\mathrm{G} 245 \mathrm{~S}}$ hot-spot mutation were detected when TIL fragments were tested against peptides and TMGs encoding patient's nonsynonymous mutations, and 3 TCRs targeting this mutation were isolated from TIL fragments (26). Here, to screen for neoantigen-reactive TILs we utilized the sorting strategy that was described in Figure 1 and Figure 2A. We sorted 3,300 cells expressing CD3 ${ }^{+} \mathrm{PD}-1^{+}$and/or CD134 (denoted with "O"), and 1,320 cells expressing CD3 ${ }^{+} \mathrm{PD}-1^{+}$and/or $\mathrm{CD} 137^{+}$(denoted with "B") and cultured them at 3 cells/well in REP conditions. Three weeks later, growth efficiencies for CD $3^{+} \mathrm{PD}-1^{+}$and/or CD134 ${ }^{+}$ and $\mathrm{CD} 3^{+} \mathrm{PD}-1^{+}$and/or $\mathrm{CD} 137^{+}$were $12 \%$ and $7 \%$, respectively. In addition to identifying $\mathrm{CD} 4^{+} \mathrm{T}$ cells that recognized 3 patient-specific neoantigens that were not detected with the standard TIL fragment screening method (Supplemental Figure 2), our new culture method also detected multiple microcultures that recognized the TP53 ${ }^{\mathrm{G} 245 \mathrm{~S}}$ hot-spot mutation (Figure 4, A and B).

To further characterize the TP53 $3^{\mathrm{G} 245 \mathrm{~S}}$-reactive TCRs, we first sequenced the TCRs from cultures O37, O71, and O102, using 5'RACE. The $\alpha$ and $\beta$ chains were subcloned into a retroviral plasmid and transduced into allogeneic donor PBMCs. To determine the HLA restriction of the TCRs, we coincubated the TCRtransduced PBMCs with COS7 cells transiently expressing the patient's HLA class II molecules and pulsed with mutated TP53 peptide. Interestingly, the recognition of all 3 TCRs was restricted to HLA-DRB3*02:02 

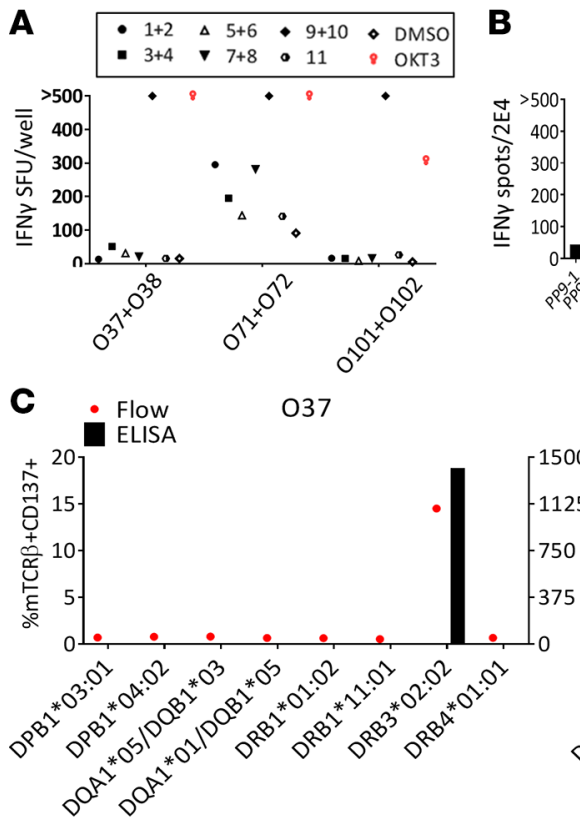

D

O37

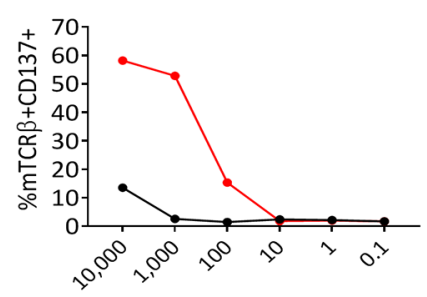

B

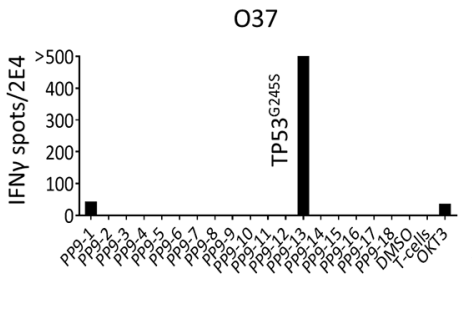

071

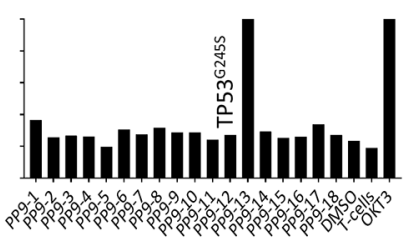

071

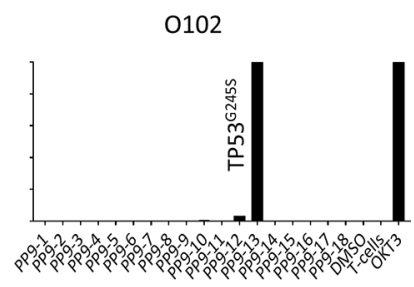

0102
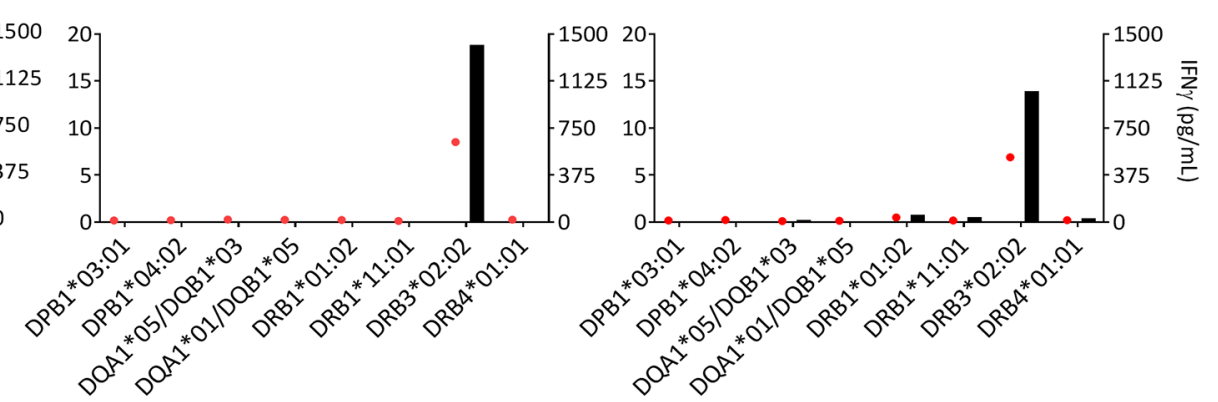

0102
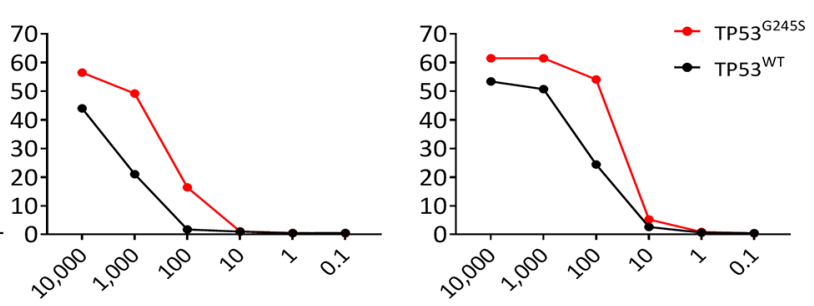

Figure 4. Isolation and characterization of 3 TP53 ${ }^{\mathrm{C} 2455}$ neoantigen-reactive TCRs from Pt.4127 sorted TIL microwell cultures. TILs from tumor digest were sorted and expanded based on the expression of PD-1 and/or CD134. (A) Cells from 2 cultures were combined and cocultured with DCs that were pulsed with pools of mutated peptides. Showing cultures displayed enhanced IFN- $\gamma$ secretion against peptide pools 9 and 10 (pool 9 encompassing TP53 mutated peptide) in (B) IFN- $\gamma$ ELISPOT assay at 16 hours. Following expansion, $2 \times 10^{4}$ cells from individual TIL cultures that showed specific reactivity against peptide pool 9 (not shown) were cocultured with $1 \times 10^{5} \mathrm{DCs}$ pulsed with single peptides present in the pool. (C and D) TCRs targeting TP53 2455 were sequenced, synthesized, and virally delivered into allogeneic PBMCs to assess reactivity and specificity. (C) TCR-transduced cells were coincubated with COS7 cells that were transfected with plasmids encoding the patient's HLA class II and pulsed with mutated 25mers. (D) Cells were coincubated with autologous DCs pulsed with serially diluted TP53 $3^{\mathrm{C} 2455}$ and WT peptides. ' $>$ ' denotes greater than 500 spots. All data are representative of at least 2 independent experiments except in $\mathbf{A}$.

(Figure 4C), which is expressed in up to $33 \%$ of White individuals in the US (www.allelefrequencies.net). Finally, to test the avidity of recognition, we cocultured TCR-transduced cells with autologous DCs pulsed with a serial dilution of mutated and WT peptides (Figure 4D). Although all 3 TCRs had higher avidity for the mutated peptide compared with the WT peptide, the TCR isolated from the O37 culture showed greater specificity against the mutated peptide compared with the other 2 TCRs. Due to the high allele frequency of HLA-DRB3 ${ }^{*} 02: 02$, the frequency of the mutation ( $2.8 \%$ of all tumors; see ref. 27 ), and high specificity of the receptor, the TCR isolated from $\mathrm{O} 37$ might be attractive as an off-the-shelf reagent that could be used in TCR-gene therapies for patients with metastatic cancer. Altogether, using our high-throughput method we were able to detect and isolate multiple TCRs from $\mathrm{CD} 4^{+}$cells targeting 4 neoantigens. Three of these neoantigens were not detected when TIL fragments were tested.

Isolation of a highly potent KRAS $S^{G I 2 V}$-targeting TCR. Encouraged by our ability to isolate TCRs targeting the TP53 ${ }^{\mathrm{G} 245 \mathrm{~S}}$ hot-spot mutation and given that KRAS mutations, mainly at positions 12 and 13 , are highly prevalent $(28,29)$, we sought to use our high-throughput culturing approach to identify neoantigenreactive $\mathrm{T}$ cells in tumors expressing KRAS driver mutations. For this purpose, we used cryopreserved tumor digest from Pt.4148 to prepare the microwell cultures. Pt.4148, a metastatic endometrial cancer patient, was enrolled in NCT01174121 and her tumor TIL fragments were screened for neoantigen reactivities. No reactivity was found against the peptide pools or against the KRAS ${ }^{\mathrm{G} 12 \mathrm{~V}} 24 \mathrm{mer}$, which was pulsed 

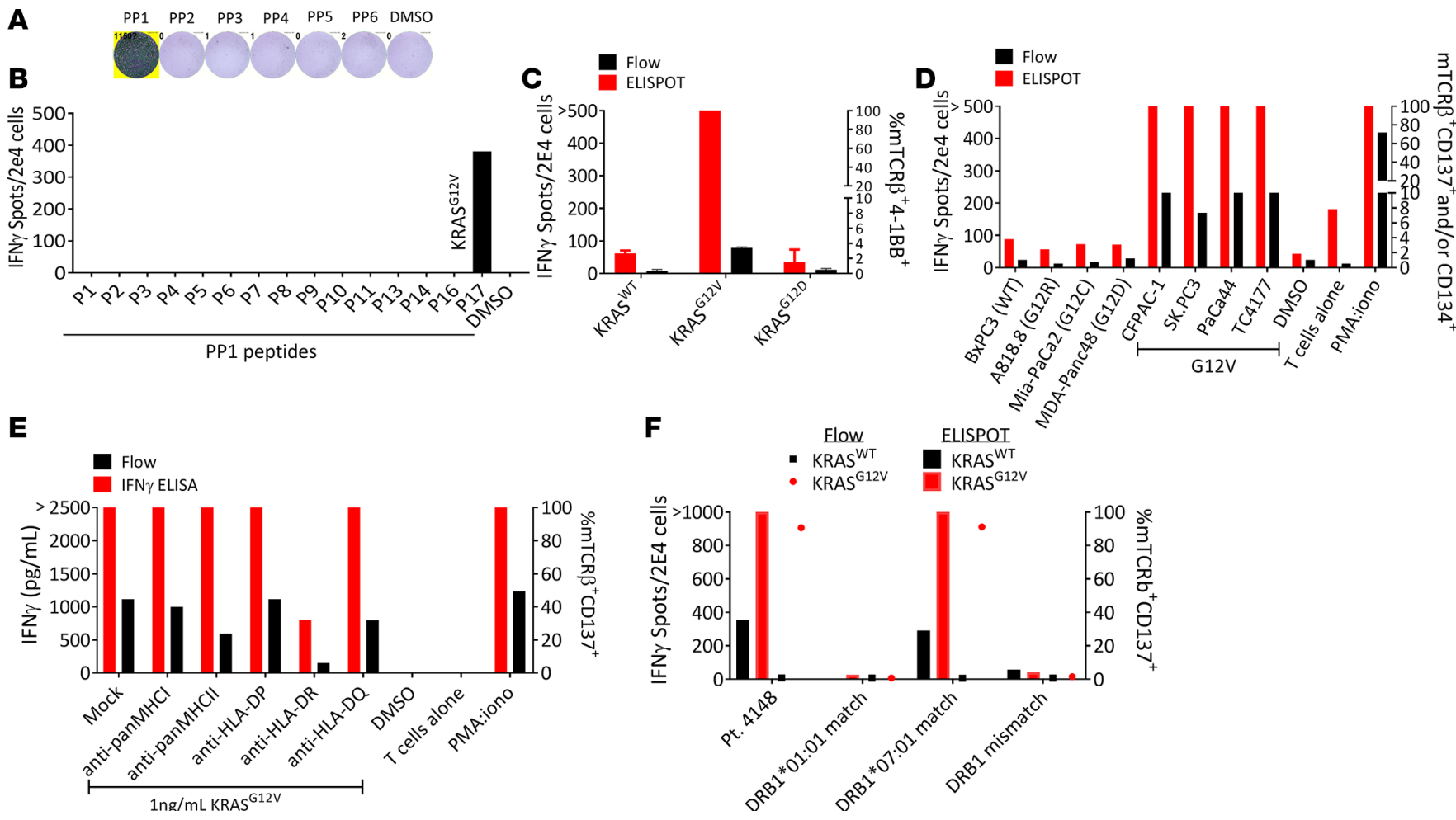

Figure 5. Characterization of a highly potent HLA-DRB1*07:01-restricted TCR isolated from a metastatic lesion of endometrial cancer. $C D 3^{+} P D-1^{+}$and/ or CD134+ $4^{+}$TLs were sorted, expanded at 3 cells/well, and cultures that grew were tested. (A) TIL microwell culture that showed recognition against DCs pulsed with pooled peptide pools (PP) were expanded and IFN- $\gamma$ secretion was assessed following coculture for $16-20$ hours with DCs pulsed with single peptide pools, and (B) single peptides from PP1. (C and $\mathbf{D})$ The functionality of autologous PBMCs virally transduced with the TCR isolated from neoantigen-reactive culture was measured following incubation with (C) DCs liposomally transfected with full-length RNA encoding for KRAS ${ }^{\text {WT }}$, KRAS ${ }^{\mathrm{C} 12 \mathrm{~V}}$, and KRAS ${ }^{G 12 D}$, and (D) DCs loaded with supernatant from lysed cell lines that underwent 5 cycles of freezing and thawing at 1:5:10 ratio (T cells/DCs/cell lines). (E) Autologous DCs pulsed with the mutated peptide were incubated with HLA-blocking antibodies for 2 hours prior to the addition of the PBMCs expressing the TCR. (F) Effector cells expressing the TCRs were incubated with DCs (pulsed with the mutated peptide) from donors matched at one of the DRB1 alleles or with DCs from a complete DRB1 mismatch. '>' denotes greater than 500 spots. All data are representative of at least 3 independent experiments.

individually in the screen (data not shown). Therefore, we used our high-throughput screening method to test whether we could identify neoantigen-reactive T cells. We sorted $1,720 \mathrm{CD}^{+} \mathrm{PD}-1^{+}$and/or CD $134^{+}$ TIL cells, expanded them at 3 cells/well, and 76 cultures were screened 3 weeks later $(\sim 13.5 \%$ growth efficiency) against 6 peptide pools (Supplemental Table 4). Only 1 microwell CD4 ${ }^{+}$culture, W7, demonstrated enhanced IFN- $\gamma$ secretion when tested against peptide pools (Figure 5A). Deconvolution of the peptides from PP1 showed PP1-17, a 24mer peptide encompassing the KRAS ${ }^{\mathrm{G} 12 \mathrm{~V}}$ mutation, as the potential neoantigen targeted by W7 (Figure 5B). The TCR from culture W7 TIL microwell culture was Sanger sequenced and revealed unique $\alpha$ and $\beta$ chains that were subcloned into a retroviral expression plasmid and transduced into autologous PBMCs for further testing. Interestingly, the TCR sequence was present at a very low frequency $(0.056 \%)$ in the tumor digest and ranked 287 based on TCR $\beta$ deep sequencing.

In order to test the specificity of the receptor, autologous DCs were liposomally transfected with RNA expressing full-length WT KRAS, KRAS ${ }^{\mathrm{G} 12 \mathrm{D}}$, or $\mathrm{KRAS}^{\mathrm{G} 12 \mathrm{~V}}$, washed, and cocultured with transduced PBMCs expressing the receptor. Both cell surface upregulation of CD137, assessed by flow cytometry, and IFN- $\gamma$ secretion demonstrated high specificity of KRAS ${ }^{\mathrm{G} 12 \mathrm{~V}}$ recognition (Figure $5 \mathrm{C}$ ). Since the TCR was isolated from $\mathrm{CD}^{+}$cells, the TCR more likely recognized a mutated protein that was presented by MHC II molecules expressed by APCs that had taken up the antigen from apoptotic cancer cells in the tumor microenvironment or draining lymph nodes. Thus, to test whether this TCR could recognize tumor lysates derived from $\mathrm{KRAS}^{\mathrm{G} 12 \mathrm{~V}}$-expressing cancer cells, we cocultured TCR-transduced cells with autologous DCs loaded with supernatant from cell lysates originating from mutated-KRAS-expressing cell lines (5:1 ratio of DC/cell lines) including the TC4177, $\mathrm{KRAS}^{\mathrm{G} 12 \mathrm{~V}}$-expressing line established from a pancreatic lesion in our laboratory. KRAS ${ }^{\mathrm{G} 12 \mathrm{~V}} \mathrm{TCR}$-transduced cells demonstrated specific recognition against autologous DCs loaded with lysates from $\mathrm{KRAS}^{\mathrm{G} 12 \mathrm{~V}}$-expressing cell lines but not $\mathrm{KRAS}^{\mathrm{G} 12 \mathrm{D}}, \mathrm{KRAS}^{\mathrm{G} 12 \mathrm{C}}$, 
$\mathrm{KRAS}^{\mathrm{G} 12 \mathrm{R}}$ or WT, as demonstrated in Figure 5D. Furthermore, coincubation of gene-engineered T cells expressing the receptors with autologous DCs pulsed with a serial dilution of the 24 mer peptide demonstrated coreceptor-independent activation of $\mathrm{CD}^{+}$cells (Supplemental Figure 3, C and D).

To determine which HLA allele presented the mutated KRAS ${ }^{\mathrm{G} 12 \mathrm{~V}}$ epitope, we incubated autologous DCs loaded with KRAS ${ }^{\mathrm{G} 12 \mathrm{~V}}$ peptides in the presence of HLA-blocking antibodies and then performed cocultures with autologous peripheral blood lymphocytes (PBLs) transduced with the TCR. Although blocking HLA-II with anti-pan class II antibody slightly reduced CD137 expression, blocking HLA-DR showed a substantial reduction in IFN- $\gamma$ secretion and CD137 cell-surface expression (Figure 5E). To determine the specific allele that presented the $\mathrm{KRAS}^{\mathrm{G} 12 \mathrm{~V}}$ peptide, we pulsed the 24 mer peptide on DCs from donors expressing either one of the patient's HLA-DRB1 alleles or donor DCs with complete allele mismatch; then, the DCs were washed and coincubated with TCR-transduced PBMCs. A specific T cell reactivity was seen when the TCR-transduced T cells were cocultured with the HLA-DRB1*07:01 donor (Figure $5 \mathrm{E}$ ). To further test our results, and to test the possibility that $K R A S$-mutated peptide might be presented on HLA-DRB3-5 molecules, we pulsed the 24mer peptides on HEK293T-CIITA cells transfected with HLA-DRB1*07:01 and cocultured the cells with T cells expressing the TCR. Indeed, T cells were specific against the HLA-DRB1*07:01 (Supplemental Figure 3E). Further, to evaluate the potential of this potent TCR to serve as an off-the-shelf agent for cancer immunotherapy, we first assessed the expression frequencies of $\mathrm{KRAS}^{\mathrm{G} 12 \mathrm{~V}}$ and HLA-DRB1*07:01 in the US population. Based on the Allele Frequency Net Database (http://www.allelefrequencies.net) (30), in White and African Americans, 25\%-30\% and 12.6\% of the individuals express this allele, respectively. $\mathrm{KRAS}^{\mathrm{G} 12 \mathrm{~V}}$ hot-spot driver mutations are expressed at high frequency in various histologies, approximately $22 \%$ of pancreatic cancer patients (COSMIC database, http://cancer.sanger.ac.uk/cosmic); thus, given the frequencies of HLA-DRB1*07:01 and G12V among cancer patients, this TCR would be a useful candidate for an off-the-shelf cancer immunotherapy reagent. In summary, using our high-throughput sensitive approach, we were able to isolate a potent HLA class II-restricted TCR targeting the shared $\mathrm{KRAS}^{\mathrm{G} 12 \mathrm{~V}}$ mutation.

\section{Discussion}

In this study, we describe a nonbiased, high-throughput approach to identify and isolate neoantigen-reactive clones from tumor digests. Previous studies have shown that neoantigen-reactive $\mathrm{CD} 4^{+}$and $\mathrm{CD} 8^{+} \mathrm{T}$ cells can be detected in tumor lesions $(4,5,14,17,31)$ and that reinfusion of ex vivo expanded neoantigenreactive TILs can mediate tumor regression in metastatic cancer patients $(4,28,32,33)$. Although ACT of metastatic melanoma patients using unselected TILs achieved a $24 \%$ CR rate and $56 \%$ ORR (3), currently, the success in common epithelial cancers is limited. We have sought to improve the detection of neoantigen-reactive cells and shorten the time to identification of reactive TCRs for possible use in autologous cell gene therapy. By employing our new high-throughput approach we identified $\mathrm{T}$ cell reactivities against 14 new unique neoantigens that were missed when TIL fragments of the patients were screened using our standard approach.

In previous studies, several methods have been developed to identify and isolate tumor- or neoantigenreactive TILs $(12,14,15,17-19,31,34)$. However, a number of factors can limit their efficiency. The frequency of neoantigen-reactive TILs can decline dramatically when cells are expanded ex vivo as a bulk culture, possibly due to overgrowth of younger, nonreactive TILs at the expense of exhausted reactive cells. Thus, reactive T cells present at low frequency in the excised tumor, as we showed in Pt.4078, Pt.4148, and Pt.4127, may be missed in conventional functional TIL screens using fragment cultures. Additionally, the antigen-experienced Temra cells, that have very low or no expression of PD-1 (19, 22), might be missed in approaches that use the PD-1 as the sole marker for enrichment. Here, to overcome these obstacles we first FACS-isolated T cells expressing PD-1 and/or T cell activation markers from tumor digests and expanded them at low cell concentrations. The metastatic lesions studied in this work were excised from patients and tumor fragments were also cultured in high-dose IL-2 and tested against mutated peptides and TMGs, as previously described $(5,28,33)$. The remainder of the excised tumors were enzymatically digested and frozen for further testing described in this study.

We first tested our limiting-dilution approach on a tumor digest from Pt.4078. Our standard screening of TIL fragments from Pt.4078 did not reveal reactivity against the pools of the mutated 25 mer peptides (Supplemental Figure 1A). However, when sorted TILs were cultured at 3 cells/well and expanded, 13\% of the cultures grew and could be tested. To test all of these cultures against peptide pools we had to minimize 
our screening assay to a manageable size by pooling 2 microwell cultures and testing against DCs pulsed with 2 peptide pools. As demonstrated in Figure 2B, the functional assays of the mixed cultures showed enhanced IFN- $\gamma$ secretion against several peptide pools. As a control, we tested bulk FACS-isolated cells using the same gating strategy (Figure $2 \mathrm{~A}$ ) and did not find reactivity against the pools. Five unique neoantigens were discovered when TILs were cocultured with DCs loaded with individual peptides (Figure 2D), and Sanger sequencing revealed that all neoantigen-reactive cultures were clonal. To determine the frequency of neoantigen-reactive T cells in the tumor we compared these TCR sequences to sequences of bulk, $\mathrm{PD}-1^{+}$, and PD-1- tumor-resident $\mathrm{T}$ cells. Interestingly, 4 TCRs were present in very low frequency in bulk T cells and 3 of these 4 TCRs were enriched in PD- $1^{+}$sorted cells, although still found at low frequencies (Table 1). The TCR sequence from culture W8 was present in PD-1 $1^{+}$only and not in the bulk nor $\mathrm{PD}-1^{-}$. Finally, the TCR from culture W7 was present in $\mathrm{PD}-1^{-}$and the unselected bulk population but not in $\mathrm{PD}-1^{+}$, suggesting that neoantigen-reactive $\mathrm{T}$ cells can be found in the $\mathrm{PD}-1^{-} \mathrm{CD} 134^{+}$population. These results are consistent with our previous findings that neoantigen-reactive $\mathrm{T}$ cells are enriched in $\mathrm{PD}-1^{+} \mathrm{T}$ cells $(18,19)$. However, some cultures, such as W7, can be missed, suggesting that the additional markers used in our approach (CD134 or CD137) are able to improve the detection and isolation of neoantigenreactive $\mathrm{T}$ cells. To summarize, our 2-step high-throughput approach allowed us to detect $5 \mathrm{~T}$ cell clones targeting 5 different neoantigens in this patient that were missed when TILs grown from tumor fragments were screened by our conventional method.

We presumed that starting microwell cultures with a low number of antigen-experienced cells and further expanding them, using an antibody stimulation, will drive them to a more senescent state. To test our assumption, we evaluated the expression of differentiation and inhibitory markers in cells from 6 cultures of 3 patients. Five out of 6 cultures showed high expression of LAG3 and TIM-3, consistent with our assumption (Supplemental Figure 6). We thus hypothesized that reactive TCRs introduced to autologous PBLs can be more advantageous than using expanded microwell cultures for adoptive cell transfer. Here, we showed that coupling a nested scPCR step with our screening step allowed us to isolate and sequence neoantigen-reactive TCRs quickly and efficiently.

Targeting driver oncogenes using off-the-shelf therapeutic reagents is attractive since these mutations are likely to be homogeneously expressed in cancer cells across different cancer histologies (35). We recently showed that ACT using CD8 ${ }^{+}$TILs targeting the KRAS ${ }^{\mathrm{G} 12 \mathrm{D}}$ mutation led to a significant antitumor clinical response (4). Here, employing our approach on tumor samples from 2 patients enabled us to identify and isolate 4 TCRs targeting driver hot-spot mutations. Three TCRs were detected in a tumor sample from Pt.4127 targeting TP53 $3^{\mathrm{G} 245}$ in the context of HLA-DRB3*02:02 (Figure 4C). All 3 TCRs were specific against mutated TP53 peptide and not the WT counterpart. Since the frequency of TP53 ${ }^{\mathrm{G} 245 \mathrm{~S}}$ is $2.8 \%$ among all cancer patients and can be higher in several histologies (36), and the frequency of individuals expressing the HLA-DRB3*02:02 is high (16.4\% allele frequency in the White US population, http:// allelefrequencies.net), these TCRs are attractive candidates for off-the-shelf cancer immunotherapy treatments using autologous gene-engineered PBMCs.

Similar to TP53, mutations in KRAS are frequent and important for tumorigenesis of many cancers. The hot-spot mutations in KRAS occur mainly at positions 12, 13, and 61 (28). In human cancers, KRAS mutations have been identified in approximately $90 \%$ of pancreatic ductal adenocarcinoma (PDAC) (37), and $33 \%$ in colorectal cancer (COSMIC database, http://cancer.sanger.ac.uk/cosmic). In this study, we detected and isolated TCR from CD4 ${ }^{+}$TILs targeting KRAS ${ }^{\mathrm{G} 12 \mathrm{~V}}$. The G12V mutation is the second most frequent mutation among KRAS mutations $(37 \%, 35 \%, 30 \%$, and $24 \%$ in ovarian, prostate, pancreatic, and colorectal, respectively [COSMIC database]) after KRAS ${ }^{\mathrm{G} 12 \mathrm{D}}$. This TCR is a putative candidate to serve as an offthe-shelf therapeutic agent because of its high specificity for the mutated but not the WT protein (Figure $5 \mathrm{C})$. Furthermore, to evaluate the potency of the isolated TCR we incubated autologous DCs with supernatant of lysed cell lines expressing KRAS mutations followed by incubation with TCR-expressing PBLs. Although it is possible that tumor cells may express MHC class II on their surface, the predominant axis of class II-restricted peptide presentation in the tumor microenvironment likely remains APCs (38). The results of this experiment, as presented in Figure 4D, in addition to the observation that the TCR can be triggered in a coreceptor-independent manner (Supplemental Figure 3C), demonstrate a high potency of the TCR. Finally, we determined that the recognition is restricted by HLA-DRB1*07:01 using HLA-DRB1 matching donors (Figure 5E) and verified by HLA transfection into HEK293T-CIITA cells (Supplemental Figure 3D). The frequency of individuals that have HLA-DRB $1 * 07: 01$ is relatively high in the US $(25 \%-30 \%$ and $12.6 \%$ 
in White and African American, respectively). Thus, this TCR could be used as an off-the-shelf reagent for TCR-gene therapy. In summary, our new high-throughput approach for sorting and culturing TILs enabled us to isolate a rare (present at $0.056 \%$ of the rearranged TCRs in the tumor) and potent $\mathrm{KRAS}^{\mathrm{G} 12 \mathrm{~V}}$-targeting TCR from a tumor digest. This approach can complement the identification of a KRAS-targeting receptor raised by immunizing HLA transgenic mice, as reported by other members of our group (39). A previous study showed that TGB6F1 mice, which harbor the $\mathrm{KRAS}^{\mathrm{G} 12 \mathrm{R}}$ mutation in their germline, are prone to develope papilloma that progress to cancer, and immunization with the G12R peptide can generate specific CD4 reactivities. Interestingly, the generation of the $\mathrm{G} 12 \mathrm{R}$-specific $\mathrm{CD} 4^{+}$cells was correlated with enhancement of tumor growth (40). However, since the mutation was encoded in the germline there is a possibility that the immunization generated CD4 ${ }^{+}$Treg cells that promoted the tumor growth.

Our findings showed enhanced detection of neoantigen-reactive TILs. In 2 patients, Pt.4078 and Pt.4148, we isolated reactive cells that were present at a very low frequency in the tumor and were missed in TIL fragment screens (Figures 2 and 5). In the tumor sample from Pt.4127, in addition to reactivity against TP53 ${ }^{\mathrm{G} 245 \mathrm{~S}}$ that was found in TIL fragments we detected 3 additional neoantigen reactivities (Figure 4 and Table 2). In the remaining samples, although we found the same number of neoantigen reactivities as found in TIL fragments, the screen revealed several different neoantigens (Table 2). As mentioned earlier, only a small portion of the tumor was used as a source of TIL microwell cultures (while the bigger part was used to generate fragments [NCT01174121, protocol 10-C-0166]), we speculate that utilizing our approach on a bigger tumor sample may allow detecting additional reactivities. Furthermore, 2 of the neoantigen reactivities that were detected by TIL fragment screening that were not detected in our method were CD8 ${ }^{+} \mathrm{T}$ cell epitopes. There are several possible reasons we are missing the reactivities: (a) high-dose IL-2 and OKT3 are not optimal for growing CD8 ${ }^{+}$TILs in microwell cultures in REP conditions, (b) due to the small number of cells in tumor digests, in patients 4166 and 4217 we used CD134 alone in conjunction with PD-1 for cell isolation; however, several studies have reported that CD137 is a better marker for CD8 cells $(41,42)$, (c) CD4 ${ }^{+}$and CD8 ${ }^{+}$cells were not separated in the sort nor the culture and $\mathrm{CD} 4^{+}$cells may have overgrown in mixed cultures ( 3 cells/ well), and (d) in the first screen using our approach we pulse the cells with a large pool of peptides in order to minimize the assay; this strategy can reduce the sensitivity of the screen due to HLA peptide competition. To improve our approach we are planning to address these points by using larger tumor samples to generate tumor digests, separating $\mathrm{CD}^{+}$and $\mathrm{CD}^{+}$cells, using additional $\mathrm{T}$ cell activation markers for enrichment, and utilizing robotics in our pipeline to upgrade our high-throughput capacity.

In summary, we have developed a method that allows an enhanced and rapid identification of neoantigen-reactive TCRs for personalized TCR-gene therapy as well as the identification of TCRs targeting hot-spot neoantigens that may be used as off-the-shelf reagents for TCR gene therapy.

\section{Methods}

Patients, tumor biopsies, and PBMCs. All samples were obtained from patients enrolled in NIH protocol 10-C-0166 (NCT01174121) and after written, informed consent was granted. One or more metastatic lesions were resected and was used for (a) whole-exome and RNA sequencing, (b) plating 24 TIL fragments for future screening and treatment as part of the clinical protocol (11), and (c) the rest of the resected tumor was enzymatically digested followed by mechanical tissue separation, as previously described (19). Briefly, tumor specimens were minced under sterile conditions, followed by enzymatic digestion (RPMI-1640 with L-glutamine [Lonza]), $1 \mathrm{mg} / \mathrm{ml}$ collagenase IV (Sigma-Aldrich), $30 \mathrm{U} / \mathrm{ml}$ DNAse (Genentech), and antibiotics for several hours at $37^{\circ} \mathrm{C}$ and intermittent mechanical tissue separation using gentleMACS (Miltenyi Biotec). Tumor digests were then cryopreserved for further analysis. Leukapheresis was performed to obtain PBMCs.

Whole-exome and RNA sequencing. Genomic DNA purification, library construction, exome capture of approximately 20,000 coding genes, next-generation sequencing of fresh tumor embedded in optimum cutting temperature (OCT, Sakura Finetek), and a matched normal leukapheresis sample were performed as previously described (43). An mRNA sequencing library was prepared from fresh tumors using the Illumina TruSeq RNA library prep kit. Putative nonsynonymous mutations were defined by 3 or more exome variant reads, $7 \%$ or greater variant allele fraction (VAF) in the exome, and 10 or more reads in the matched normal sample. Putative mutations with a variant allele frequency of greater than $10 \%$ in the tumor exome, as well as mutations that were identified in both transcriptome and exome analyses, were initially selected for screening. 
$T$ cell sorting and in vitro expansion. Cell sorting was carried out using the FACSJazz or FACSAria IIu instrument (BD Biosciences). Tumor digests were rested overnight at $37^{\circ} \mathrm{C}$ in complete $50 / 50$ media (1:1 mix of RPMI-1640 with L-glutamine and AIMV [Gibco] supplemented with $100 \mathrm{U} / \mathrm{ml}$ penicillin, $100 \mu \mathrm{g} /$ $\mathrm{ml}$ streptomycin, $12.5 \mathrm{mM}$ HEPES, and 5\% human serum) in the absence of cytokines. Bulk-sorted cells were gated on live (PI negative), single cells, $\mathrm{CD}^{+} \mathrm{PD}-1^{+}$and/or activation markers (CD134, CD137) as shown in Figure 1. Sorted cells were diluted and seeded in 96-well plates at 3 cells/well in REP media in the presence of $50 \mathrm{ng} / \mathrm{ml}$ OKT3 (Miltenyi Biotec), 3,000 IU/ml IL-2 (Aldesleukin, Chiron), and $1 \times 10^{5}$ irradiated allogeneic feeder cells (Figure 1). On days 7 and 14, half of the medium was replaced with fresh 50/50 medium containing IL-2. Microwell cultures were tested 3-4 weeks following the initial sort.

Antibodies for flow cytometry and blocking experiments. The following fluorescently conjugated antibodies were purchased from BD Biosciences: CD3-PE/APC-H7/FITC (SK7), CD8-APC/PE-Cy7/PE (SK1), CD4-PE/FITC/BV421 (SK3), CD137-APC/PE (4B4-1), CD134-FITC/PE (ACT35), mTCRß-FITC/PE (H57-597), CD14-PE (M甲P9), CD80-PE (L307.4), and HLA-DR-PE-Cy7 (L243). Antibody PD-1-APC/ PE (MIH4) was from eBioscience. For HLA-blocking experiments we used pan-class II (clone IVA12), panclass I (clone W6/32), HLA-DR (clone HB55), HLA-DP (clone B7/21), and HLA-DQ (clone SPV-L3).

IFN- $\gamma$ ELISPOT cocultures and flow cytometry assays to assess T cell activation. For peptide presentation, we used $5 \times 10^{4}$ to $1 \times 10^{5}$ cells/well of autologous DCs as APCs. Crude peptides were used in the primary screens and HPLC-grade peptides were used in the late validation tests (GenScript). Briefly, DCs were pulsed overnight with $1-20 \mu \mathrm{g} / \mathrm{ml}$ peptides at $37^{\circ} \mathrm{C}$ in complete DC media composed of RPMI containing $5 \%$ human serum, $100 \mathrm{U} / \mathrm{ml}$ penicillin and $100 \mu \mathrm{g} / \mathrm{ml}$ streptomycin, $2 \mathrm{mM} \mathrm{L}$-glutamine, $800 \mathrm{IU} / \mathrm{ml}$ GM-CSF (Leukine), and $200 \mathrm{U} / \mathrm{ml}$ IL-4 (Peprotech). Subsequently, DCs were washed and coincubated overnight with $2 \times 10^{4} \mathrm{~T}$ cells in antibody-precoated ELISPOT plates.

For IFN- $\gamma$ ELISPOT assays, ELIIP plates (Millipore, MAIPSWU) were pretreated with $50 \mu 1$ of $70 \%$ ethanol per well, washed 3 times with PBS, and then coated with $50 \mu 1$ of $10 \mu \mathrm{g} / \mathrm{ml}$ IFN- $\gamma$ capture antibody (Mabtech, clone 1-D1K) and incubated overnight at $4^{\circ} \mathrm{C}$. For OKT3 controls, wells were coated with a mixture of IFN- $\gamma$ capture antibody and OKT3 $(1 \mu \mathrm{g} / \mathrm{ml})$. For the PMA/ionomycin control we added 1:500 diluted Cell Stimulation Cocktail (eBioscience, 00-4970-03) to T cell cultures. Prior to coculture, the plates were washed 3 times with PBS, followed by blocking with 50/50 media for 2 hours at room temperature. After 16-20 hours of coculture, cells were harvested from the ELISPOT plates into a standard 96-well round-bottom plate for flow cytometry analysis, and then the ELISPOT plates were washed 5 times with PBS plus $0.05 \%$ Tween 20 (PBS-T), and incubated for 2 hours at room temperature with $100 \mu 1 /$ well of a $0.22-\mu \mathrm{m}$-filtered $1 \mu \mathrm{g} / \mathrm{ml}$ biotinylated anti-human IFN- $\gamma$ detection antibody solution (Mabtech, clone 7-B6-1, diluted in $1 \times$ PBS supplemented with $0.5 \%$ FBS). The plate was then washed 3 times with PBST, followed by a 1-hour incubation with $100 \mu 1$ well of streptavidin-ALP (Mabtech, diluted 1:3,000 with above diluent). The plate was then washed 5 times with plain PBS followed by development with $100 \mu 1 /$ well of $0.45-\mu \mathrm{m}$-filtered BCIP/NBT substrate solution (KPL, Inc.). The reaction was stopped by rinsing thoroughly with tap water. ELISPOT plates were scanned and counted using an ImmunoSpot plate reader and associated software (Cellular Technologies Limited, Ltd). Expression of the T cell activation markers CD134 and CD137 was assessed by flow cytometry at approximately 18-24 hours after stimulation. Briefly, cells that were harvested from the ELISPOT plate were pelleted, washed with FACS buffer $(1 \times$ PBS supplemented with $1 \%$ FBS and $2 \mathrm{mM}$ EDTA), and then stained with the appropriate antibodies for 30 minutes, at $4^{\circ} \mathrm{C}$ in the dark. Cells were washed with FACS buffer prior to acquisition on a BD FACSCanto I, BD FACSCantoII, and BD LSRFortessa flow cytometers. All data were gated on live (PI negative), single cells. Data analysis was carried out using FlowJo software (Tree Star).

Generation of autologous APCs. Monocyte-derived, immature DCs were generated from PBMCs using the plastic adherence method, as previously described (5). Briefly, apheresis samples were thawed, washed, set to $5 \times 10^{6}$ to $10 \times 10^{6}$ cells $/ \mathrm{ml}$ with neat AIM-V media (Life Technologies) and then incubated at approximately $1 \times 10^{6} \mathrm{cells} / \mathrm{cm}^{2}$ in an appropriately sized tissue culture flask and incubated at $37^{\circ} \mathrm{C}, 5 \%$ $\mathrm{CO}_{2}$. After 90 minutes, nonadherent cells were collected, and the flasks were vigorously washed with AIM$\mathrm{V}$ media, and then incubated with AIM-V media for another 60 minutes. The flasks were then vigorously washed again with AIM-V media and then the adherent cells were incubated with DC media. On day 4 or 5 , DCs were collected and used or frozen for future use.

$H L A$ blocking and HLA restriction mapping. In antibody-blocking experiments, we preincubated target cells with $20-50 \mu \mathrm{g} / \mathrm{ml}$ blocking antibodies for $2-3$ hours at $37^{\circ} \mathrm{C}$, followed by coculture with effector 
T cells. 16-22 hours later, supernatants were harvested and IFN- $\gamma$ secretion was analyzed by ELISA. Cells were collected, labeled, and analyzed by flow cytometry. HLA restrictions were determined by transfecting COS7 or HEK293T-CIITA cell lines with pCDNA3.1 plasmids encoding the individual HLAs of interest. The next day, cells were washed and pulsed with mutated peptide for 2-4 hours. Subsequently, peptides were washed and effector $\mathrm{T}$ cells were added. IFN- $\gamma$ secretion was assessed by ELISPOT or quantified by ELISA. At the end of the coculture, cells were collected, labeled, and expression of CD134 and CD137 was assessed by flow cytometry.

TCR sequencing. For 5'RACE-based amplification, T cells were pelleted from TIL microwell cultures and total RNA isolated (RNeasy Mini kit, Qiagen). Total RNA then underwent 5'RACE as directed by the manufacturer (SMARTer RACE cDNA amplification kit, Clontech) using TCR $\alpha$ and $-\beta$ chain constant primers. The sequences of the $\alpha$ and $\beta$ chain constant chain primers are: $\alpha, 5$-GCCACAGCACTGTTGCTCTTGAAGTCC; $\beta$, 5'-CAGGCAGTATCTGGAGTCATTGAG. PCR products were then isolated by standard agarose gel electrophoresis and gel extraction (Clontech). Products were then either directly sequenced or TOPO-TA subcloned followed by Sanger sequencing of individual colonies (Macrogen).

scPCR for TCR sequencing was performed as previously described (31). Briefly, single-cell sorting was performed using a modified FACSAria IIu instrument based on T cell activation markers following coculture. Cells were sorted into reverse transcription PCR (RT-PCR) buffer and a series of 2 nested PCRs were done to amplify TCR $\alpha$ and $-\beta$ chains. The first RT and amplification reaction was performed with a One-Step RT-PCR kit (Thermo Fisher Scientific) using multiplex PCR with multiple V $\alpha$ and V $\beta$ region primers and one primer for $\mathrm{C} \alpha$ and $\mathrm{C} \beta$ regions each. The RT-PCR reaction was performed according to the manufacturer's instructions using the following cycling conditions: $50^{\circ} \mathrm{C}, 15$ minutes; $95^{\circ} \mathrm{C}, 2$ minutes; $95^{\circ} \mathrm{C}, 15$ seconds, $60^{\circ} \mathrm{C}, 4$ minutes $\times 18$ cycles; $4^{\circ} \mathrm{C}$. For the second amplification reaction, $3 \mu 1$ from the first RT-PCR was used as a template in total $25 \mu \mathrm{l}$ PCR mix using HotStarTaq DNA polymerase (Qiagen) and multiple internally nested $\mathrm{V} \alpha$ and $\mathrm{V} \beta$ region primers and 1 internally nested primer for $\mathrm{C} \alpha$ and $\mathrm{C} \beta$ regions each (final concentration of each primer was $0.6 \mu \mathrm{M}$ ). The cycling conditions were $95^{\circ} \mathrm{C}, 15 \mathrm{~min}$ utes; $94^{\circ} \mathrm{C}, 30$ seconds, $50^{\circ} \mathrm{C}, 30$ seconds, $72^{\circ} \mathrm{C}, 1$ minute $\times 50$ cycles; $72^{\circ} \mathrm{C}, 10$ minutes; $4{ }^{\circ} \mathrm{C}$. The PCR products were purified and sequenced by the Sanger method with internally nested $\mathrm{C} \alpha$ and $\mathrm{C} \beta$ region primers by GenScript.

TCR-V $\beta$ deep sequencing was performed by immunoSEQ (Adaptive Biotechnologies) on genomic DNA isolated from TIL microwell cultures or frozen tumor.

TCR construction and viral transduction. Construction of the TCRs was done by fusing the V-D-J of the $\mathrm{V} \beta$ regions to the mouse TCR $\beta$ constant chain, and the TCR $\alpha$ V-J regions to the mouse TCR $\alpha$ constant chains. The chains were separated by a furin-SGSG-P2A linker. The chains were then synthesized and subcloned into the pMSGV1 retroviral vector (GenScript).

Viral transduction was performed as described previously (5). Briefly, 2 days prior to transduction PBMCs were cultured and stimulated in 24-well plates at $2 \times 10^{6}$ cells/well in 50/50 media in the presence of $300 \mathrm{IU} / \mathrm{ml} \mathrm{IL-2}$ and $50 \mathrm{ng} / \mathrm{ml} \mathrm{OKT3}$. To generate transient retroviral supernatants, pMSGV1 encoding the TCRs $(2 \mu \mathrm{g} /$ well $)$ and the envelope-encoding plasmid pRD114 (1.4 $\mu \mathrm{g} /$ well) were cotransfected using Lipofectamine 2000 (Life Technologies) into the retroviral packaging cell line HEK293GP $\left(1 \times 10^{6}\right.$ cells per well of 6-well poly-D-lysine-coated plates, plated the day prior to transfection in the absence of antibiotics). Retroviral supernatants were collected at 48 hours after transfection, diluted 1:1 with DMEM media, and then centrifuged onto Retronectin-coated $(10 \mu \mathrm{g} / \mathrm{ml}$, Takara), non-tissue culture-treated 6-well plates at $2,000 \mathrm{~g}$ for 2 hours at $32^{\circ} \mathrm{C}$. Activated T cells $\left(2 \times 10^{6} \mathrm{cells} /\right.$ well, at $0.5 \times 10^{6} \mathrm{cells} / \mathrm{ml}$ in IL-2-containing $\mathrm{T}$ cell media) were then spun onto the retrovirus plates for 10 minutes at $300 \mathrm{~g}$ (low acceleration and brake). Activated T cells were transduced overnight, removed from the plates, and further cultured in 50/50 media containing $300 \mathrm{IU} / \mathrm{ml} \mathrm{IL}-2$. GFP and mock-transduction controls were included in transduction experiments. Cells were typically assayed 10-14 days after retroviral transduction.

Study approval. All patient samples were obtained in the course of a National Cancer Institute Institutional Review Board-approved clinical trial, and patients provided informed consent.

\section{Author contributions}

RY designed the study. RY and SAR wrote the manuscript. ET, DCD, AG, AP, MRP, and GC contributed to experiments. JJG and TDP sequenced and analyzed tumor samples. ET, DCD, AG, PFR, and SAR contributed to data analysis and interpretation. 


\section{Acknowledgments}

We thank Q. Wang and K. Hanada for providing KRAS-mutated cell lines, A. Mixon and S. Farid for flow cytometry support, and other members of the Surgery Branch for helpful discussions and technical support. This research was supported by the Center for Cancer Research intramural research program of the National Cancer Institute.

Address correspondence to: Steven A. Rosenberg, National Cancer Institute, 10 Center Drive MSC 1201, CRC Room 3-3940, Bethesda, Maryland 20892, USA. Phone: 301.496.4164; Email: sar@nih.gov.

1. Vogelstein B, Papadopoulos N, Velculescu VE, Zhou S, Diaz LA, Kinzler KW. Cancer genome landscapes. Science. 2013;339(6127):1546-1558.

2. Murphy K, Weaver C. Janeway's Immunobiology. New York, New York: Garland Science; 2016.

3. Goff SL, et al. Randomized, prospective evaluation comparing intensity of lymphodepletion before adoptive transfer of tumorinfiltrating lymphocytes for patients with metastatic melanoma. J Clin Oncol. 2016;34(20):2389-2397.

4. Tran E, et al. T-cell transfer therapy targeting mutant KRAS in cancer. N Engl J Med. 2016;375(23):2255-2262.

5. Tran E, et al. Cancer immunotherapy based on mutation-specific $\mathrm{CD} 4^{+} \mathrm{T}$ cells in a patient with epithelial cancer. Science. 2014;344(6184):641-645.

6. Stevanovi冈 S, et al. Landscape of immunogenic tumor antigens in successful immunotherapy of virally induced epithelial cancer. Science. 2017;356(6334):200-205.

7. Jiang Y, Li Y, Zhu B. T-cell exhaustion in the tumor microenvironment. Cell Death Dis. 2015;6:e1792.

8. McGranahan N, Swanton C. Clonal heterogeneity and tumor evolution: Past, present, and the future. Cell. 2017;168(4):613628.

9. McGranahan N, et al. Clonal neoantigens elicit T cell immunoreactivity and sensitivity to immune checkpoint blockade. Science. 2016;351(6280):1463-1469.

10. Tran E, Rosenberg SA. T-cell therapy against cancer mutations. Oncotarget. 2014;5(13):4579-4580.

11. Tran E, et al. Immunogenicity of somatic mutations in human gastrointestinal cancers. Science. 2015;350(6266):1387-1390.

12. Cohen CJ, et al. Isolation of neoantigen-specific $\mathrm{T}$ cells from tumor and peripheral lymphocytes. J Clin Invest. 2015;125(10):3981-3991.

13. Gros A, et al. Prospective identification of neoantigen-specific lymphocytes in the peripheral blood of melanoma patients. Nat Med. 2016;22(4):433-438.

14. Robbins PF, et al. Mining exomic sequencing data to identify mutated antigens recognized by adoptively transferred tumorreactive T cells. Nat Med. 2013;19(6):747-752.

15. Parkhurst $\mathrm{M}$, et al. Isolation of T-cell receptors specifically reactive with mutated tumor-associated antigens from tumor-infiltrating lymphocytes based on CD137 expression. Clin Cancer Res. 2017;23(10):2491-2505.

16. Martin SD, Wick DA, Nielsen JS, Little N, Holt RA, Nelson BH. A library-based screening method identifies neoantigen-reactive T cells in peripheral blood prior to relapse of ovarian cancer. Oncoimmunology. 2017;7(1):e1371895.

17. Linnemann $\mathrm{C}$, et al. High-throughput epitope discovery reveals frequent recognition of neo-antigens by $\mathrm{CD} 4^{+} \mathrm{T}$ cells in human melanoma. Nat Med. 2015;21(1):81-85.

18. Inozume $\mathrm{T}$, et al. Selection of $\mathrm{CD} 8^{+} \mathrm{PD}-1^{+}$lymphocytes in fresh human melanomas enriches for tumor-reactive $\mathrm{T}$ cells. $J$ Immunother. 2010;33(9):956-964.

19. Gros A, et al. PD-1 identifies the patient-specific CD $8^{+}$tumor-reactive repertoire infiltrating human tumors. J Clin Invest. 2014;124(5):2246-2259.

20. Seliktar-Ofir S, et al. Selection of shared and neoantigen-reactive T cells for adoptive cell therapy based on CD137 separation. Front Immunol. 2017;8:1211.

21. Kansy BA, et al. PD-1 status in CD8 ${ }^{+} \mathrm{T}$ cells associates with survival and anti-PD-1 therapeutic outcomes in head and neck cancer. Cancer Res. 2017;77(22):6353-6364.

22. Larbi A, Fulop T. From "truly naïve" to "exhausted senescent" T cells: when markers predict functionality. Cytometry A. 2014;85(1):25-35.

23. Klebanoff CA, Gattinoni L, Restifo NP. CD8 $8^{+}$T-cell memory in tumor immunology and immunotherapy. Immunol Rev 2006;211:214-224.

24. Ye Q, et al. CD137 accurately identifies and enriches for naturally occurring tumor-reactive T cells in tumor. Clin Cancer Res. 2014;20(1):44-55.

25. Tran E, Robbins PF, Rosenberg SA. 'Final common pathway' of human cancer immunotherapy: targeting random somatic mutations. Nat Immunol. 2017;18(3):255-262.

26. Deniger DC, et al. T-cell responses to TP53 "hotspot" mutations and unique neoantigens expressed by human ovarian cancers [published online ahead of print May 31, 2018]. Clin Cancer Res. https://doi.org/10.1158/1078-0432.CCR-18-0573.

27. Freed-Pastor WA, Prives C. Mutant p53: one name, many proteins. Genes Dev. 2012;26(12):1268-1286.

28. McCormick F. KRAS as a therapeutic target. Clin Cancer Res. 2015;21(8):1797-1801.

29. Jinesh GG, Sambandam V, Vijayaraghavan S, Balaji K, Mukherjee S. Molecular genetics and cellular events of K-Ras-driven tumorigenesis. Oncogene. 2018;37(7):839-846.

30. González-Galarza FF, et al. Allele frequency net 2015 update: new features for HLA epitopes, KIR and disease and HLA adverse drug reaction associations. Nucleic Acids Res. 2015;43(Database issue):D784-D788.

31. Pasetto A, et al. Tumor- and neoantigen-reactive T-cell receptors can be identified based on their frequency in fresh tumor. Cancer Immunol Res. 2016;4(9):734-743. 
32. Prickett TD, et al. Durable complete response from metastatic melanoma after transfer of autologous T cells recognizing 10 mutated tumor antigens. Cancer Immunol Res. 2016;4(8):669-678.

33. Goff SL, et al. Recognition of autologous neoantigens by tumor infiltrating lymphocytes derived from breast cancer metastases. Cancer Res. 2017;77(4_Suppl):P2-04-02.

34. Lu YC, et al. An efficient single-cell RNA-Seq approach to identify neoantigen-specific T cell receptors. Mol Ther. 2018;26(2):379-389.

35. Kandoth C, et al. Mutational landscape and significance across 12 major cancer types. Nature. 2013;502(7471):333-339.

36. Bouaoun L, et al. TP53 variations in human cancers: new lessons from the IARC TP53 database and genomics data. Hum Mutat. 2016;37(9):865-876.

37. Lennerz JK, Stenzinger A. Allelic ratio of KRAS mutations in pancreatic cancer. Oncologist. 2015;20(4):e8-e9.

38. Zanetti M. Tapping CD4 T cells for cancer immunotherapy: the choice of personalized genomics. J Immunol. 2015;194(5):2049-2056.

39. Wang QJ, Yu Z, Griffith K, Hanada K, Restifo NP, Yang JC. Identification of T-cell receptors targeting KRAS-mutated human tumors. Cancer Immunol Res. 2016;4(3):204-214.

40. Siegel CT, et al. Enhanced growth of primary tumors in cancer-prone mice after immunization against the mutant region of an inherited oncoprotein. J Exp Med. 2000;191(11):1945-1956.

41. Croft M. The role of TNF superfamily members in T-cell function and diseases. Nat Rev Immunol. 2009;9(4):271-285.

42. Lee HW, Park SJ, Choi BK, Kim HH, Nam KO, Kwon BS. 4-1BB promotes the survival of CD8 ${ }^{+} \mathrm{T}$ lymphocytes by increasing expression of Bcl-xL and Bfl-1. J Immunol. 2002;169(9):4882-4888.

43. Jones $\mathrm{S}$, et al. Frequent mutations of chromatin remodeling gene ARID1A in ovarian clear cell carcinoma. Science. 2010;330(6001):228-231. 